\title{
THE ORGANISATION OF THE INCOMPATIBILITY FACTORS IN HIGHER FUNGI: THE EFFECT OF STRUCTURE AND SYMMETRY ON BREEDING
}

\author{
JUDITH STAMBERG and Y. KOLTIN \\ Genetics Unit, Faculty of Life Sciences, Tel-Aviv University, Tel-Aviv, Israel
}

Received 16.ii.72

\begin{abstract}
SUMmary
An evaluation of the structure of incompatibility systems in the higher fungi and its effect on inbreeding and outbreeding indicates that: In unifactorial systems a one-locus structure, which permits 50 per cent. inbreeding, is preferable to a two-locus structure, which would permit inbreeding to rise above 50 per cent. In bifactorial systems a two-locus structure for each factor is advantageous because it makes possible a high outbreeding potential with a small number of alleles. The two-locus structure also confers flexibility on the inbreeding potential. A symmetrical distribution of alleles among the four loci of a two-locus bifactorial system allows for maximal outbreeding. The advantage of symmetry is most pronounced when the total number of allelic specificities is not large. The asymmetrical distribution of alleles among the four incompatibility loci of Schizophyllum commune suggests that its incompatibility system evolved from a one-locus unifactorial system. The $A \beta$ locus, with 32 alleles, is assumed to be the oldest locus; the remaining three loci, with 9 alleles each, are assumed to have come into operation within a short time of each other, at a later stage.
\end{abstract}

\section{Introduction}

IN the higher fungi (Homobasidiomycetes) sexual dimorphism does not occur, but the great majority of species are heterothallic and sexual reproduction is rigorously controlled by incompatibility genes. These genes serve as a means of recognition between strains, and permit complete sexual morphogenesis to occur only if the two strains carry unlike incompatibility specificities.

Two types of incompatibility systems are found: unifactorial heterothallism and bifactorial heterothallism. In the former, a single incompatibility factor (called $A$ ) determines mating competence. The progeny of a cross between strains carrying allelic specificities $A 1$ and $A 2$, for example, are of the two parental types, hence the unifactorial system has been termed "bipolar". A large number of factor specificities exists in nature; in Polyporus betulinus, 28 specificities have been found (Burnett, 1965), in $P$. palustris about 20 are estimated to exist (Flexer, 1965), and in Fomes cajanderi about 33 (Neuhauser and Gilbertson, 1971).

In the bifactorial systems two incompatibility factors, called $A$ and $B$, together control mating competence. Two strains are completely compatible only if they have different specificities for each factor. In all species examined, these factors are genetically unlinked, and their independent segregation at meiosis leads to the production of four main classes of progeny, hence the term "tetrapolar" has been applied to this system (e.g. a cross between strains carrying $A 1 B 1$ and $A 2 B 2$ produces equal numbers of 
progeny carrying $A 1 B 1, A 1 B 2, A 2 B 1$ and $A 2 B 2)$. Each incompatibility factor possesses a large number of specificities (Fries and Jonasson, 1941; Whitehouse, 1949; Raper, Krongelb and Baxter, 1958; Prévost, 1962; Day, 1963).

The incompatibility factors in bifactorial systems are characteristically composed of two loci each, designated $\alpha$ and $\beta$. This was first demonstrated for the $A$ factor of Schizophyllum commune (Papazian, 1951), and has since been shown to be the case for the $B$ factor of this species (Raper, Baxter and Middleton, 1958; Koltin, Raper and Simchen, 1967), as well as for other species (Day, 1960; Terakawa, 1960; Takemaru, 1961). In every case the component loci of a factor are linked and separable by recombination. The specificity of an incompatibility factor is determined by the allelic specificities of its component loci. Every combination of an $\alpha$ allele and a $\beta$ allele leads to a new specificity; for example, $A \alpha 1-\beta 1$ has a different $A$ factor specificity from $A \alpha 1-\beta 2, A \alpha 2-\beta 1$ and $A \alpha 2-\beta 2$. Each of the component loci possesses a large number of natural specificities. In $S$. commune, which has been the most extensively studied among the higher fungi, the number of alleles found in nature has been estimated at 9 for the $A \alpha$ locus (Raper, Baxter and Ellingboe, 1960), 32 for $A \beta$ (see appendix of the present paper) and 9 each for $B \alpha$ and $B \beta$ (Parag and Koltin, 1971). Thus approximately 288 (i.e. $9 \times 32$ ) different $A$ factor specificities and 81 different $B$ factor specificities $(9 \times 9)$ can exist in nature.

The incompatibility factors of unifactorial fungi, however, fail to reveal evidence of a two-locus structure, although such evidence has been intensively sought in two species (Flexer, 1963; Burnett, unpublished, quoted by Raper, 1966).

The rôle of the incompatibility systems of higher fungi in controlling the potential for inbreeding and outbreeding has been assessed by various authors. Mather (1942) and later Whitehouse (1949) pointed out that a unifactorial system with multiple specificities would permit 50 per cent. inbreeding and a level of outbreeding proportional to the number of specificities in the population. They noted that the introduction of a second factor would decrease inbreeding, and that multiple specificities for each factor would serve to retain a high outbreeding potential.

Papazian (1951), who first demonstrated the two-locus structure of an incompatibility factor in a bifactorial fungus, realised the advantage that such a structure would confer on both the inbreeding and outbreeding potential of a species. A cross between strains carrying $A \alpha 1-\beta 1$ and $A \alpha 2-\beta 2$ would produce recombinants $A \alpha 1-\beta 2$ and $A \alpha 2-\beta 1$ in low frequency; such recombinants would be compatible with both parental factors as well as with each other and hence the inbreeding potential would be increased by an amount proportional to the linkage intensity. Under certain conditions an increase in inbreeding would be advantageous, and in general the inbreeding potential would increase only slightly because of tight linkage between the loci. The advantage conferred by a two-locus structure on outbreeding would be very great, because a single mutation at one of the two component loci would produce not just one new factor specificity but a whole series of new specificities by recombination, which would eventually pair every $\alpha$ allele in the population with every $\beta$ allele. Simchen (1967), whose mathematical treatment supports Papazian's interpretation, pointed out an additional advantage to the two-locus structure, in that a small 
population can maintain a large number of potential incompatibility factor specificities, and the probability of loss of a specificity is thus very low.

We wish to consider here the effects of the unifactorial and bifactorial systems on breeding, in light of our current knowledge of the structure of the incompatibility factors and the number of specificities found in natural populations. This consideration suggests the probable direction of evolution of the breeding systems of higher fungi.

\section{UNIFAGTORIAL SYSTEM: WHY ONLY ONE LOCUS PER FAGTOR?}

As stated above, any one progeny of a cross in a unifactorial species is compatible with 50 per cent of its sibs. If the factor, which we can call $A$, were composed of two loci, $\alpha$ and $\beta$, the inbreeding potential would increase to a level determined by the frequency of recombination between the loci. If the two loci were unlinked the inbreeding potential would be 75 per cent.; if linked, the inbreeding potential would be between 50 and 75 per cent., according to the formula $50 \%+P_{A}-P_{A}^{2}$, where $P_{A}=$ frequency of recombination between the component loci.

The outbreeding potential, i.e. the probability that any two unrelated strains will be compatible, is determined by the number of specificities possessed by the incompatibility factor. In a unifactorial system with only two specificities, the outbreeding potential will be 50 per cent. With four specificities the outbreeding potential would rise to 75 per cent., assuming that the specificities are randomly distributed in the population; with 20 specificities the outbreeding potential reaches 95 per cent., as calculated from the formula $\left(n_{A}-1\right) / n_{A}$, where $n_{A}=$ number of factor specificities (Raper, 1966).

If the factor were composed of two loci, the outbreeding potential would vary according to the formula $\left(n_{A_{\alpha}} n_{A_{\beta}}-1\right) / n_{A_{\alpha}} n_{A \beta}$, where $n_{A_{\alpha}}$ and $n_{A \beta}$ are the number of allelic specificities at the two component loci. With two alleles at each locus the total number of factor specificities is four $(2 \times 2)$, and the outbreeding potential is 75 per cent.; with five alleles at each locus the total number of factor. specificities is 25 and the outbreeding potential is 96 per cent. If 20 alleles are distributed equally between two loci, 100 factor specificities result and the outbreeding potential reaches 99 per cent. Thus, a higher outbreeding potential is attained by distributing a given number of allelic specificities between two loci rather than by retaining all the specificities at a single locus (fig. 1). With a large number of alleles, however, the difference in outbreeding potential is slight.

A two-locus unifactorial system would have, therefore, a slight increase in its outbreeding potential and an increase in its inbreeding potential from 50 per cent. to a maximum of 75 per cent., depending on the linkage relationship between the loci (fig. 3). The advantage of the former would appear to be offset by the disadvantage of the latter, as no two-locus unifactorial species is known.

\section{Bifagtorial SYSTEM: WHy TWO LINKED LOGI PER FAGTOR?}

As Mather (1942) noted, the introduction of a second incompatibility factor would immediately lower the inbreeding potential from 50 to 25 per cent. The outbreeding potential would also be severely decreased, but this 
effect would be eliminated by the accumulation of a large number of specificities for the second factor.

For example, if a second incompatibility factor, which can be called $B$, comes into operation in a unifactorial species with 20 specificities for its first factor, the inbreeding potential drops to 25 per cent. (fig. 3). The outbreeding potential drops from 95 per cent. to a level determined by the number of specificities at the second factor, according to the formula $\left(n_{A} n_{B}-n_{A}-n_{B}+1\right) / n_{A} n_{B}$, where $n_{A}$ and $n_{B}$ are the number of specificities in the first and second factor, respectively. With only two specificities at the $B$ factor the outbreeding potential is 47.5 per cent. To regain the 95 per cent. outbreeding potential permitted by the original unifactorial

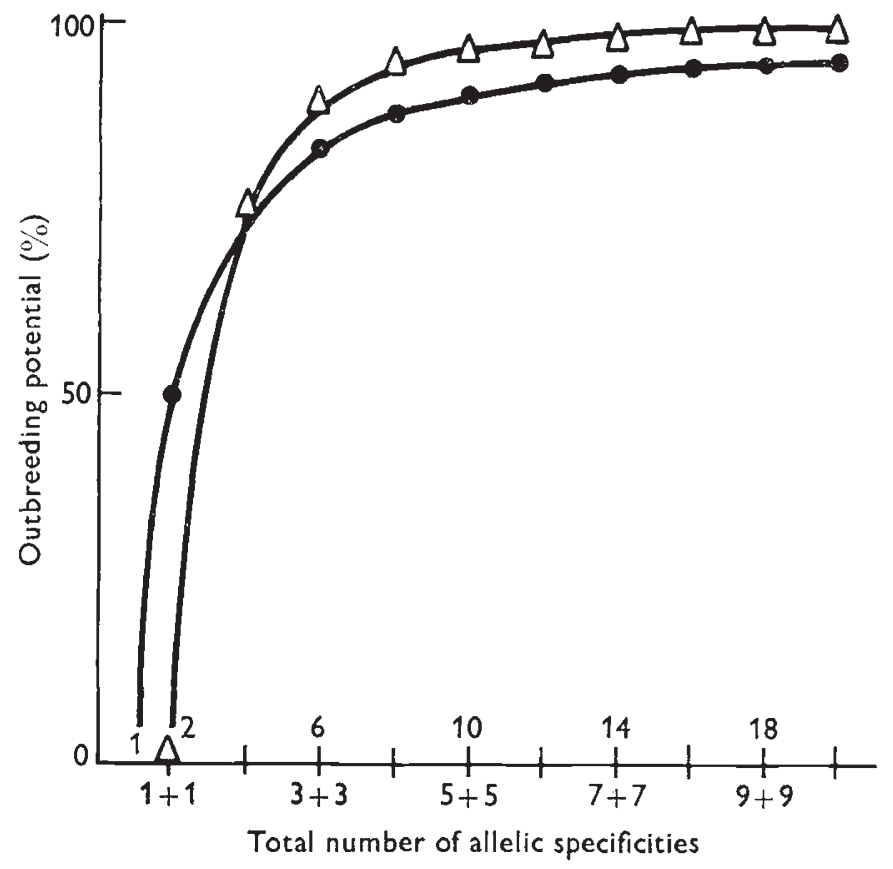

FIG. 1.-Outbreeding potential in one-locus and two-locus unifactorial systems. $0=1$ locus system. $\triangle=2$-locus system. For the 2-locus system it is assumed that the allelic specificities are distributed equally between the two loci.

structure would require the accumulation of more than 30 additional specificities at each factor, which represents a total increment of more than sixty mutational events (fig. 2).

(Throughout this discussion it is assumed that the original locus continues to produce new allelic specificities at the same rate as do new loci. This may be a questionable assumption since presumably only a finite number of functional alleles can be produced at any locus. However, even if it is assumed that the original locus produces no new allelic specificities, the conclusions remain the same.)

If a second locus becomes operative in the new incompatibility factor, fewer mutational events are required in order to regain the original outbreeding potential. (The addition of a new locus to the older factor instead 
of the younger would contribute very little, as the older factor already possesses a large number of specificities.)

If the original locus continues to accumulate allelic specificities at the same rate as do the new loci (which can be called $B \alpha$ and $B \beta$ ), then the original 95 per cent. level would be reached upon accumulation of nine new alleles at each locus, as calculated from the formula

$$
\left(n_{A} n_{B_{\alpha}} n_{B \beta}-n_{A}-n_{B_{\alpha}} n_{B \beta}+1\right) / n_{A} n_{B_{\alpha}} n_{B \beta},
$$

where $n_{A}, n_{B_{\alpha}}, n_{B_{\beta}}$ are the number of specificities for the original factor and the two component loci of the new factor, respectively. Thus about 30 mutational events can restore outbreeding to the 95 per cent. level.

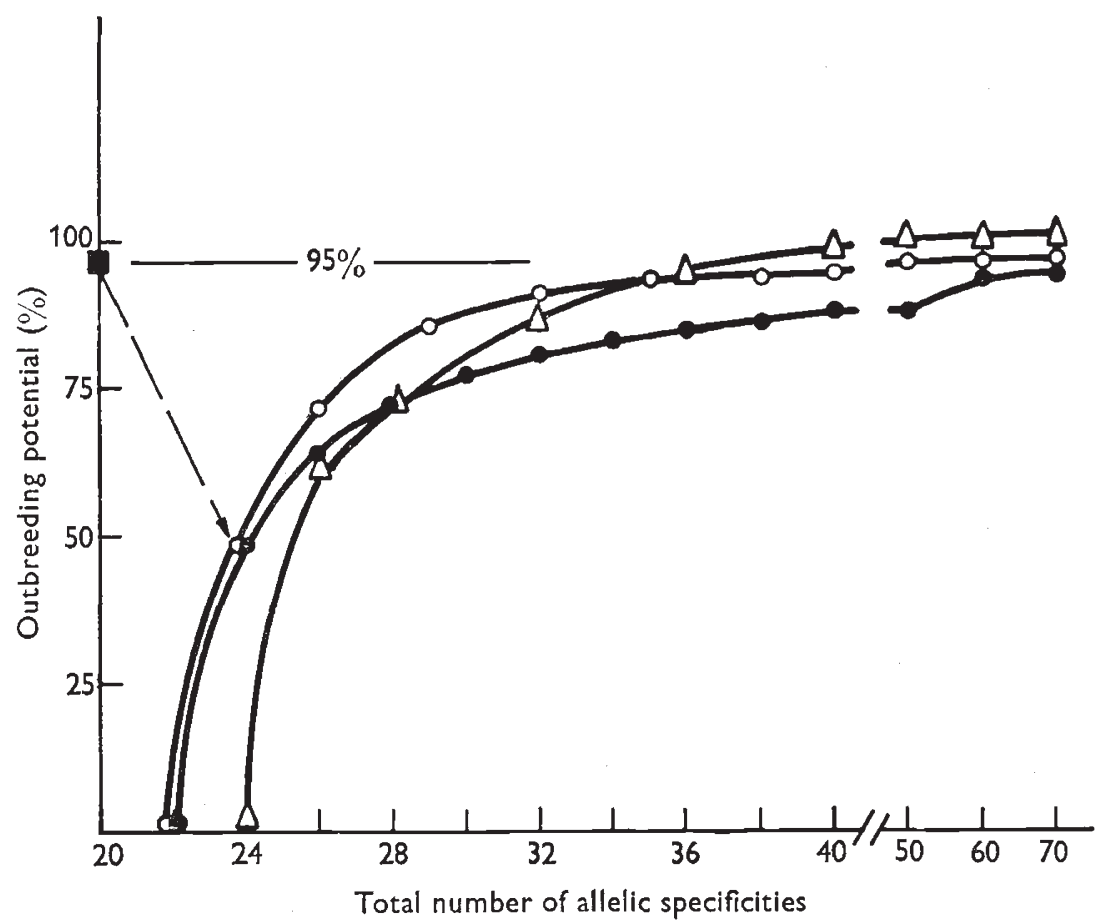

FIG. 2.-Outbreeding potential in bifactorial systems in which none, one, or both of the factors have two loci. $=$ each factor has one locus. $\mathrm{O}=$ the original factor has one locus; the new factor has two loci. $\triangle=$ each factor has two loci. It is assumed that the original factor had 20 allelic specificities, and continued to accumulate new specificities at the same rate as did the new loci. $\quad(\square=$ the outbreeding potential in the original unifactorial system, included for comparison.)

The addition of the second locus to the new factor would increase the inbreeding potential from the 25 per cent. possible in a one-locus two-factor system up to a maximum of 37.5 per cent., if the three loci were unlinked (fig. 3). Linkage between the two loci of the new factor would reduce the inbreeding potential to a value between these extremes.

An additional economy of mutational events would be achieved if a second locus were added to the original factor as well as to the new factor. Accumulation of 20 mutations, divided equally among the four loci, would bring the outbreeding potential to the original 95 per cent. level, as calculated 
from the formula $\left(n_{A \alpha} n_{A \beta} n_{B_{\alpha}} n_{B_{\beta}}-n_{A \alpha} n_{A \beta}-n_{B_{\alpha}} n_{B_{\beta}}+1\right) / n_{A \alpha} n_{A \beta} n_{B_{\alpha}} n_{B_{\beta}}$, where $n_{A_{\alpha}}, n_{A \beta}, n_{B_{\alpha}}$ and $n_{B_{\beta}}$ are the number of specificities at the original locus and the three new loci, respectively (fig. 2).

The inbreeding potential in such a system (fig. 3) is increased from 25 per cent. to a level determined by the linkage relationships of the component loci of each factor, according to the formula $\frac{1}{4}\left(1+2 P_{A}-2 P_{A}^{2}\right)\left(1+2 P_{B}-2 P_{B}^{2}\right)$, where $P_{A}$ and $P_{B}$ are the frequencies of recombination between the component loci of the $A$ and $B$ factor, respectively (Simchen, 1967). If all four loci were unlinked, the inbreeding potential would be 56 per cent. In all the bifactorial fungi examined, however, the $A$ and $B$ factors are unlinked but the $\alpha$ and $\beta$ comprising each factor are linked. The highest recorded values for recombination frequencies are 35 per cent. in the $A$

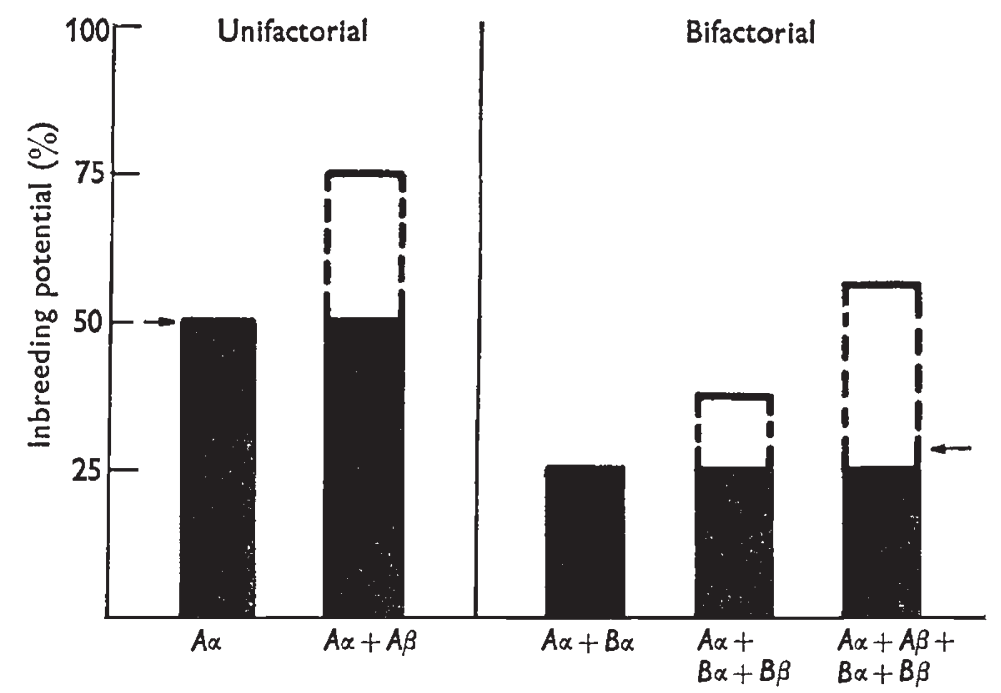

FiG. 3.--Inbreeding potential in unifactorial and bifactorial systems. Unshaded areas indicate the range of inbreeding permitted in systems with a two-locus structure, where linkage between the loci determines the exact level of inbreeding. Arrows indicate the actual inbreeding potential of those unifactorial and bifactorial species studied.

factor of Schizophyllum commune in one cross and 11.6 per cent. in the $B$ factor of the same species in another cross (Stamberg, 1968); these frequencies, occurring in one cross, would permit 44 per cent. inbreeding. The average frequency of $A$ factor recombination in a random sample of crosses taken from worldwide locations is 9.45 per cent. (data from Raper, Baxter and Ellingboe, 1960); the average $B$ factor recombination frequency is 2.3 per cent. (Koltin, Raper and Simchen, 1967). Recombination at these frequencies would permit 30.6 per cent. inbreeding. Independent data, obtained by Schaap and Simchen (1971), show the inbreeding potential of a natural population of $S$. commune to be fixed (by the recombination frequencies in the $A$ and $B$ factors) at 25-29 per cent. Thus the inbreeding potential of $S$. commune can realistically be estimated at $25-30$ per cent. The scant data available in other bifactorial species (Takemaru, 1961) indicate that the inbreeding potential is less than 34 per cent. and usually lower. 
The two-locus structure confers an additional advantage, that of flexibility, on the inbreeding potential. In $S$. commune the frequencies of recombination between the two loci of each factor are genetically controlled and can vary, according to the genetic background and the environment, within a range of tenfold or more (Simchen, 1967; Stamberg, 1968; Stamberg and Simchen, 1970). Low recombination is dominant to high (Simchen, 1967; Stamberg, 1969); in addition, the controlling alleles for low recombination in both incompatibility factors are found more frequently in nature than are the alleles for high recombination (Stamberg, 1969). Recombination is thus kept low, and inbreeding is therefore generally kept near the 25 per cent. level; yet the species possesses the competence for higher recombination frequencies, hence a higher inbreeding potential, under certain conditions where this may be advantageous.

Thus, in the bifactorial system a two-locus structure allows maximal outbreeding with a given number of allelic specificities, and linkage between the two loci confers flexibility of inbreeding. A great advantage is obtained by a two-locus structure of the new factor; a smaller advantage, which may or may not be significant, accrues with the introduction of a second locus in the original factor (fig. 2). Studies on the structure of various bifactorial systems may well show that in some fungi only one factor is composed of two loci. Indications that this may be the case are discussed by Raper (1966). In such fungi, the one-locus factor can be assumed to be the original factor.

\section{Distribution of SPECIFICITIES IN BIFAGTORIAL SYSTEMS: THE EFFECT OF SYMMETRY}

The existence of multiple alleles at each of the four incompatibility loci comprising the bifactorial system implies the gradual accumulation of products of mutational events. Their distribution among the four loci has a profound effect on the outbreeding potential (fig. 4).

Consider a hypothetical pool of 20 mutational events, each producing a different allelic specificity. If these were distributed equally among the four loci, the outbreeding potential would be 92 per cent. Any deviation from this complete symmetry results in a lowering of the outbreeding potential. If the $A$ and $B$ factors each retain 10 of these 20 allelic specificities, but the distribution within each factor is asymmetrical, for example $8 \mathrm{A \alpha}$, $2 A \beta, 8 B \alpha$ and $2 B \beta$, the outbreeding potential drops; in the example given here the value is 88 per cent. If the distribution within each factor is symmetrical but the two factors have unequal numbers of specificities, e.g. $8 A \alpha, 8 A \beta, 2 B \alpha$ and $2 B \beta$, the outbreeding potential drops; in this example it is 74 per cent. Asymmetry both between and within factors, e.g. $14 A \alpha$, $2 A \beta, 2 B \alpha$ and $2 B \beta$, allows an outbreeding potential of 72 per cent. Thus the most economical situation, i.e. the distribution which provides the highest outbreeding potential from a given number of alleles, is that of complete symmetry between and within the two factors. Asymmetry within a factor results in a small loss of efficiency whereas asymmetry between the two factors results in a significant decrease in efficiency.

The advantage of symmetry is most pronounced when the total number of specificities is not large. With very many specificities, every distribution allows high outbreeding. 


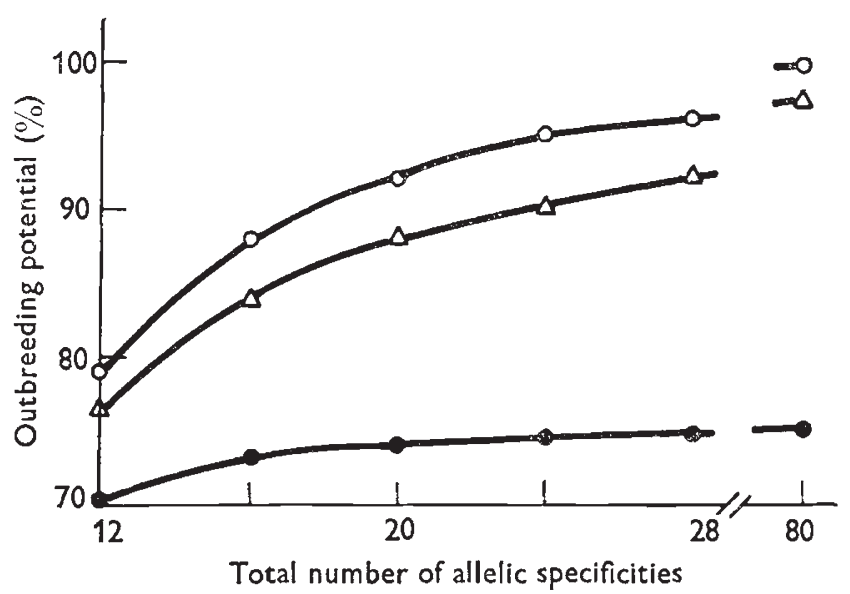

FIG. 4.-The effect of the distribution of allelic specificities, within and between factors, on outbreeding. $=$ symmetrical distribution within factors; asymmetrical distribution between factors. $\triangle=$ asymmetrical distribution within factors; symmetrical distribution between factors. $O=$ symmetrical distribution within and between factors. For the asymmetrical cases, each point represents the most extreme asymmetry possible. For example, 16 allelic specificities were distributed:

$6 A \alpha, 6 A \beta, 2 B \alpha, 2 B \beta$.

$\triangle 6 A \alpha, 2 A \beta, 6 B \alpha, 2 B \beta$.

$\bigcirc 4 A \alpha, 4 A \beta, 4 B \alpha, 4 B \beta$.

\section{SCHIZOPHYLLUM COMMUNE: A CASE OF ASYMMETRY}

As stated above, Schizophyllum commune is the most extensively analysed bifactorial species. Studies of the frequency and distribution of incompatibility alleles in a worldwide sample have led to an estimate of 9 alleles at the $A \alpha$ locus, with a range of 7 to 13 (Raper, Baxter and Ellingboe, 1960); $32 A \beta$ alleles, with a range of 23 to 59 (see appendix to this paper); and 9 alleles each at $B \alpha$ and $B \beta$, which are believed to represent the total number of natural alleles (Parag and Koltin, 1971).

The marked asymmetry both between factors (a total of 41 alleles within the $A$ factor as compared to 18 within the $B$ factor) and within one of the factors ( $9 A \alpha$ and $32 A \beta$ alleles) is in apparent contradiction to the argument developed in the preceeding section, that asymmetry is an inefficient distribution of alleles with respect to the outbreeding potential. If the twolocus bifactorial system were the ancestral structure from which the other breeding systems of the higher fungi evolved, as Raper (1966) has proposed, then one would expect a more symmetrical distribution of alleles than the one actually found. Especially when the system was new and beginning to accumulate alleles, there would be a high selective value to a symmetrical distribution of alleles, which would maximise the outbreeding efficiency.

If, however, the bifactorial system evolved from an older unifactorial system, as Whitehouse (1949) has suggested, then a quite different distribution of alleles would be anticipated. A unifactorial system would have a high inbreeding potential and also a high outbreeding potential, assuming it possessed many incompatibility alleles for its one factor. The introduction of a second incompatibility factor would immediately lower the inbreeding potential. As explained in section 3, a very large number of alleles would 
have to accumulate at both factors before the outbreeding potential were restored to its original level. With an original 20 alleles at the first factor, approximately 60 additional alleles would have to accumulate before the outbreeding level were allowed by the new bifactorial structure to reach the original 95 per cent. If a second locus were brought into operation in the new factor, only 30 new alleles would be needed before the outbreeding potential reached 95 per cent.; and if a second locus were brought into operation in the original factor as well, about 20 new alleles, distributed among the four loci, are needed to reach the 95 per cent. outbreeding level. Thus evolution from unifactorial to bifactorial implies an asymmetrical distribution of allelic specificities, with the older, original locus possessing many more specificities than the newer loci.

The evolution of the incompatibility system of $S$. commune can be visualised on this basis. Originally a unifactorial system with approximately 20 allelic specificities for its $A$ factor, it enjoyed a 95 per cent. outbreeding potential (but suffered from a 50 per cent. inbreeding level). The introduction of a second factor, $B$, lowered inbreeding to 25 per cent. but the outbreeding potential was also drastically lowered. New alleles were accumulated at both $A$ and $B$ by mutational events, but the improvement in outbreeding potential was only gradual (see fig. 2). A significant improvement in outbreeding potential was gained by the introduction of a second locus at the $B$ factor; an additional small, but perhaps significant, improvement was gained by the introduction of a second locus at the $A$ factor. The number of allelic specificities found today at each of the loci suggests that the original locus was the one known today as $A \beta$; assuming that it has continued to produce new allelic specificities from the time of its origin till the present, it can be inferred that it possessed about 20 allelic specificities at the time the $B$ factor came into operation and has since accumulated an additional ten or so alleles. The three loci $B \alpha, B \beta$ and $A \alpha$ subsequently became operative within a short time of each other, hence they each possess far fewer alleles than the older $A \beta$. Each of the three new loci possesses about the same number of alleles, since they are about the same age.

An alternative interpretation of the asymmetrical distribution of alleles in $S$. commune is that the $A \beta$ locus has an inherently greater potential for functional variation, perhaps due to a size difference. This interpretation implies that the asymmetry is fortuitous.

Analysis of the distribution of alleles in a series of distantly related species of the higher fungi would distinguish between the two interpretations. If the asymmetry in $S$. commune reflects the evolution of the system, then other bifactorial species should also exhibit the same type of asymmetry. If, however, the asymmetry in $S$. commune reflects a chance difference in the structure of the loci, then distantly related species would not exhibit a common pattern of allelic distribution.

\section{Appendrx}

$A$ re-estimate of the number of natural alleles at the $\mathrm{A} \beta$ locus of Schizophyllum commune

From an analysis of the allelic specificities found in the $A$ factors of part of a worldwide sample of strains of $S$. commune, Raper, Baxter and 
Ellingboe (1960) estimated the total number of $A \beta$ alleles in nature to be 50 , with 5 per cent. confidence limits of 32 and 91 . Their estimate was based on the number of repeats of alleles in the sample, according to the method of Dobzhansky and Wright (1941).

Examination of the data used for this estimate reveals, however, that the sample was not taken randomly from the worldwide collection. A prior analysis of this collection had shown that, within the 114 monokaryotic

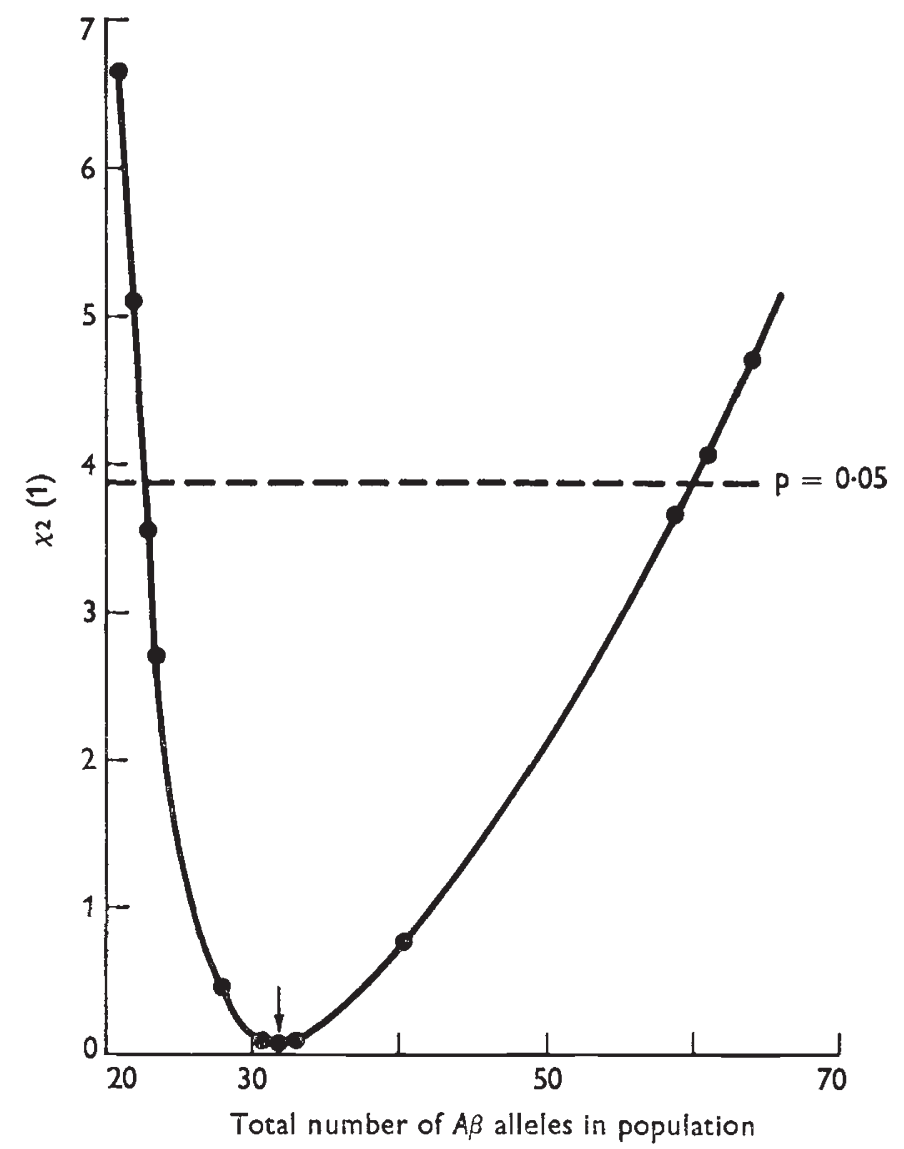

FIG. 5.-Estimation of the total number of $A \beta$ alleles of $S$. commune in the population by the minimal chi-square method. The arrow indicates the best estimate.

strains, $79 A$ factors were found once each, 16 were found twice each, and one was found three times (Raper, Krongelb and Baxter, 1958). Thus strains with non-repeated $A$ factors constituted 69 per cent. of the total sample. In the later analysis by Raper, Baxter and Ellingboe (1960) for $A \alpha$ and $A \beta$ specificities, about one-quarter of the total sample was used (32 strains of the total 114). A random sample of 32 of the 114 strains would have been made up of approximately 69 per cent. non-repeated $A$ factors and 31 per cent. repeated $A$ factors. However, the factors in the sample of 32 were selected on the basis of prior knowledge of duplication, or lack of duplication, of their $A$ factors among the sample; 13, or 41 per 
cent. of the 32 were strains known to have $A$ factors duplicated by other strains in the total population, but these duplicates were purposely excluded from the sample of 32 . Thus, this small sample does not accurately reflect the population, in which there are a number of repeated $A$ factors. The estimate of the number of $A \beta$ specificities derived from this sample, therefore, is an overestimate, since the actual number of repeats of allelic specificities in the population is greater than the number used in the calculations.

From the data given by Raper et al. (1960) the number of repeats of $A \beta$ alleles in the population was re-estimated by applying a correction factor to the number of $A \beta$ repeats among all the repeated $A$ factors; this was done to bring representation of the repeated $A$ factors into line with their frequency $(0.31)$ in the worldwide sample of 114 . The distribution obtained is only an approximation but is closer to the natural situation than the distribution estimated previously. (Since the number of $A \propto$ alleles in the sample was small, and all the expected alleles have been actually found in the sample, re-estimation of $A \alpha$ was not necessary.)

An estimate of the number of $A \beta$ alleles in nature, based on this distribution, was made by the method of minimal chi-squares (Koltin et al., 1967). This method avoids estimation of sample variance which is required for Dobzhansky and Wright's calculation; instead, the number of repetitions of alleles is assumed to fit a Poisson distribution, and a $\chi^{2}$ for goodness of fit to a Poisson distribution is calculated by entering a series of whole numbers for the number of alleles found in nature but not represented in the sample. The minimal chi-square is obtained when the total number of $A \beta$ alleles is 32 ; the range of values which do not give a significant chisquare at the 5 per cent. level is 23-59 (fig. 5).

Raper et al. (1958) estimated that approximately 339 distinct $A$ factors exist in nature. The earlier estimation of $9 A \alpha$ and $50 A \beta$ allelic specificities would give $450 A$ factors; the new estimate for $A \beta$ would allow $9 \times 32=288$ $A$ factors, which is in closer agreement to the expected number of $A$ factors.

\section{REFERENCES}

BURNETT, J. H. 1965. The natural history of recombination systems. In Incompatibility in Fungi (K. Esser and J. R. Raper, eds.), pp. 98-113. Springer-Verlag, New York.

DAY, P. R. 1960. The structure of the $A$ mating-type locus in Coprinus lagopus. Genetics, $45,641-651$.

DAY, P. R. 1963. The structure of the $A$ mating-type factor in Coprinus lagopus: wild alleles. Genet. Res., Camb., 4, 323-325.

DOBZHANSKY, T., AND WRIGHT, s. 1941. Genetics of natural populations. V. Relations between mutation rates and accumulation of lethals in populations of Drosophila pseudoobscura. Genetics, 26, 23-52.

FleXer, A. s. 1963. Bipolar incompatibility in Polyporus palustris. Thesis, Harvard Univ., Cambridge, Mass.

Flexer, A. s. 1965. Bipolar incompatibility in Polyporus palustris. (Abstr.) Amer. Jour. Bot., 52, 634 .

FRIES, N., AND JONASSON, L. 1941. Über die Interfertilität verschiedener Stamme von Polyporus abietinus. Svensk. Bot. Tidskr., 35, 177-193.

KOLTIN, Y., RAPER, J. R., AND SIMCHEN, G. 1967. The genetic structure of the incompatibility factors of Schizophyllum commune: The $B$ factor. Proc. Natl. Acad. Sci., U.S., 57, 55-62.

MATHER, K. 1942. Heterothally as an outbreeding mechanism in fungi. Nature, 149, 54-56. NEUHAUSER, K. S., AND GILBERTsON, R. L. 1971. Some aspects of bipolar heterothallism in Fomes cajanderi. Mycologia, 63, 722-735.

PAPAZIAN, H. P. 1951. The incompatibility factors and a related gene in Schizophyllum commune. Genetics, 36, 441-459. 
PARAG, Y., AND Koltin, Y. 1971. The structure of the incompatibility factors of Schizophyllum commune: Constitution of the three classes of $B$ factors. Molec. Gen. Genetics, 112, 43-48.

PRÉvost, G. 1962. Étude génétique d'un Basidiomycète: Coprinus radiatus. Thesis, Univ. de Paris, Paris.

RAPER, J. R. 1966. Genetics of Sexuality in Higher Fungi. Ronald Press, New York. viii + $283 \mathrm{pp}$.

RAPER, J. R., BAXTER, M. G., AND ELlingboe, A, H. 1960. The genetic structure of the incompatibility factors of Schizophyllum commune: The A-factor. Proc. Natl. Acad. Sci., U.S., 46, 833-842.

RAPER, J. R., BAXTER, M. G., AND MIDDLETON, R. B. 1958. The genetic structure of the incompatibility factors in Schizophyllum commune. Proc. Natl. Acad. Sci., U.S., 44, 889-900.

RAPER, J. R., KRONGElB, G. S., AND BAXTER, M. G. 1958. The number and distribution of incompatibility factors in Schizophyllum commune. Amer. Nat., 92, 221-232.

SCHAAP, T., AND SIMGHEN, G. 1971. Genetic control of recombination affecting mating factors in a population of Schizophyllum, and its relation to inbreeding. Genetics, 68, 67-75.

SIMCHEN, G. 1967. Genetic control of recombination and the incompatibility system in Schizophyllum commune. Genet. Res., Camb., 9, 195-210.

sTAmberg, J. 1968. Two independent gene systems controlling recombination in Schizophyllum commune. Molec. Gen. Genetics, 102, 221-228.

STAMBERG, J. 1969. Genetic control of recombination in Schizophyllum commune: The occurrence and significance of natural variation. Heredity, 24, 361-368.

STAMBERG, J., AND SIMCHEN, G. 1970. Specific effects of temperature on recombination in Schizophyllum commune. Heredity, 25, 41-52.

TAkemaru, T. 1961. Genetic studies on fungi. X. The mating system in Hymenomycetes and its genetical mechanism. Biol. Four. Okayama Univ., 7, 133-211.

TERAKAWA, H. 1960. The incompatibility factors in Pleurotus ostreatus. Scientific papers of the College of Gen. Education, Univ. Tokyo, 10, 65-71.

Whitehouse, H. L. K. 1949. Heterothallism and sex in fungi. Biol. Rev., 24, 411-447. 